Research article Open Access

\title{
Effect of small interference RNA (siRNA) for ADAMTS5 on intervertebral disc degeneration in the rabbit anular needle-puncture model
}

\author{
Shoji Seki ${ }^{1}$, Yumiko Asanuma-Abe ${ }^{1,2}$, Koichi Masuda ${ }^{3}$, Yoshiharu Kawaguchi ${ }^{1}$, Kunihiro Asanuma ${ }^{2}$, \\ Carol Muehleman ${ }^{4}$, Akiko Iwai ${ }^{1}$ and Tomoatsu Kimura ${ }^{1}$
}

\begin{abstract}
1Department of Orthopaedic Surgery, Faculty of Medicine, University of Toyama, 2630 Sugitani, Toyama, Toyama 930-0194, Japan 2Department of Orthopedic Surgery, Rush Medical College at Rush University Medical Center, 1653 W Congress Parkway, Chicago, IL 60612, USA ${ }^{3}$ Department of Orthopaedic Surgery, School of Medicine, University of California, San Diego, 9500 Gilman Drive, Mail Code 0863, La Jolla, CA 92093-0863, USA

${ }^{4}$ Department of Biochemistry, Rush Medical College at Rush University Medical Center, 1653 W Congress Parkway, Chicago, IL 60612, USA
\end{abstract}

Corresponding author: Shoji Seki, seki@med.u-toyama.ac.jp

Received: 21 Aug 2009 Revisions requested: 16 Sep 2009 Revisions received: 10 Oct 2009 Accepted: 4 Nov 2009 Published: 4 Nov 2009

Arthritis Research \& Therapy 2009, 11:R166 (doi:10.1186/ar2851)

This article is online at: http://arthritis-research.com/content/11/6/R166

(c) 2009 Shoji Seki et al.; licensee BioMed Central Ltd.

This is an open access article distributed under the terms of the Creative Commons Attribution License (http://creativecommons.org/licenses/by/2.0), which permits unrestricted use, distribution, and reproduction in any medium, provided the original work is properly cited.

\begin{abstract}
Introduction The etiology of degenerative disc disease is unknown. Several investigators have reported the presence of proteolytic enzymes, such as the matrix metalloproteinase (MMP) and ADAMTS (a disintegrin and metalloprotease with thrombospondin-like repeats) families, in degenerated human discs. Glasson and colleagues recently reported that a significant reduction occurs in the severity of cartilage destruction in ADAMTS5 knockout mice compared with wildtype mice. The purpose of this study was to evaluate the suppressive effects of injections of ADAMTS5 small interference RNA (siRNA) oligonucleotide on intervertebral disc degeneration in the rabbit anular needle-puncture model.

Methods Rabbit nucleus pulposus (NP) cells were transfected with siRNA oligonucleotides specific for ADAMTS5 or the control. The suppression of the ADAMTS5 gene by siRNA transfection was assessed by using real-time polymerase chain reaction ( $P C R$ ), both in monolayer and alginate bead cultures with or without interleukin-1 $\beta$ (IL-1 $\beta$ ) stimulation. The effect of siRNA was determined in vivo by using the rabbit anular needlepuncture model (control group: $n=8$; ADAMTS5 group: $n=8$ ). One week after the initial anular puncture, the animals received

an injection of the control or anti-ADAMTS5 oligonucleotide ( $100 \mu \mathrm{g}$ each at the $\mathrm{L} 2 / 3$ and $L 4 / 5$ level; 16 discs/group). Disc height, magnetic resonance imaging (MRI) (Thompson classification and signal intensity), and safranin-O staining (histologic grade) were assessed.

Results IL-1 $\beta$ treatment significantly increased the ADAMTS5 mRNA level in NP cells $(P<0.01)$. ADAMTS5 gene suppression was $70 \%$ compared with the control oligonucleotide in both monolayer and alginate bead culture with or without stimulation with IL-1 $\beta$. The injection of antiADAMTS5 oligonucleotide in vivo resulted in improved MRI scores with increased signal intensity and improved histologic grade scores with statistical significance $(P<0.05)$. No significant change in disc height was observed.

Conclusions A single injection of ADAMTS5 siRNA induced the suppression of degradation in NP tissues, as shown by significantly improved MRI and histologic grades. The mechanism of response to siRNA may be worthy of exploration for possible therapeutic purposes.
\end{abstract}

ADAMTS: a disintegrin and metalloprotease with thrombospondin-like repeats; $\mathrm{AF}$ : anulus fibrosus; $\mathrm{C}_{\mathrm{T}}$ : comparative threshold; $\mathrm{DHI}$ : disc height index; GAPDH: glyceraldehyde 3-phosphate dehydrogenase; IL-1 $\beta$ : interleukin-1 $\beta$; IVD: intervertebral disc; MMP: matrix metalloproteinase; MRI: magnetic resonance imaging; NP: nucleus pulposus; PBS: phosphate-buffered saline; PCR: polymerase chain reaction; RT-PCR: reverse transcriptasepolymerase chain reaction; siRNA: small interference RNA; TNF- $\alpha$ : tumor necrosis factor- $\alpha$. 


\section{Introduction}

The intervertebral disc (IVD) encompasses two structures, the anulus fibrosus (AF) and the nucleus pulposus (NP). The predominant matrix component of the AF is collagen type I, whereas the NP contains randomly organized collagen fibers (mainly type II) and highly hydrated proteoglycans, primarily aggrecan, which impart compressive resistance to the tissue. Aggrecan is cleaved at a specific "aggrecanase" site [1,2]; this cleavage results from the action of several members of the ADAMTS (adisintegrin and metalloprotease with thrombospondin-like repeats) family [3-6]. In a murine model of osteoarthritis, ADAMTS5-knockout mice have a significantly reduced level of cartilage destruction, compared with wildtype mice [7]. Glasson and colleagues [8] also reported that no effect in ADAMTS4 knockout mice was noted on the progression or severity of osteoarthritis after surgical induction of joint instability. However, the dual deletion of ADAMTS4 and ADAMTS5 provided significant protection against proteoglycan degradation ex vivo and decreased the severity of murine osteoarthritis in vivo [9].

Compared with cartilage, the NP has a higher content of aggrecan that is more degraded and a higher proportion of molecules not bound to hyaluronan [10]. A recent human cadaveric study revealed the presence of aggrecanase-generated aggrecan fragments and abundant levels of ADAMTS5 in human IVDs, regardless of the level of disc degeneration, based on magnetic resonance imaging (MRI) grade classification [11]. This study also showed that larger quantities of ADAMTS4 are present in human NP and AF tissues derived from discs with a greater level of disc degeneration (grade 4) compared with those from discs with lower level of disc degeneration (grade 2). Thus, it remains unclear whether ADAMTS4 or ADAMTS5 is the major aggrecanase responsible for degradation of aggrecan in the human IVD. Modulating the enzymatic activity or gene expression of the responsible enzymes might be a valid approach for protecting human IVD tissues from degradation.

IVDs of patients with lumbar disc herniation have been shown to express proinflammatory cytokines, such as interleukin-1 $\beta$ (IL-1 $\beta)$ and tumor necrosis factor- $\alpha$ (TNF- $\alpha$ ) [12,13], which are known to stimulate the expression of ADAMTS in bovine cartilage $[14,15]$. The regulation of ADAMTS4 and ADAMTS5 has been reported to differ slightly. For example, although a highly selective inhibitor of $I_{\kappa} B$ kinase did not inhibit the secretion of ADAMTS4, it blocked ADAMTS5 secretion in the same concentration range that inhibited aggrecan degradation in bovine cartilage [15]. Furthermore, whereas ADAMTS5 mRNA was expressed in human normal and OA cartilage [16], ADAMTS4 mRNA was very low in vivo and was induced in vitro only after stimulation with IL-1 $\beta$. These results suggest that investigating both ADAMTS4 and ADAMTS5 may shed light on the mechanism of IVD degeneration.
Biologic treatment strategies for human IVD degeneration include increasing the levels of anabolic growth factors or blocking the catabolic cascade or both. On the anabolic side, an in vivo rabbit anular puncture model of disc degeneration showed the anabolic effects of bone morphogenetic protein-7 [17] and growth and differentiation factor-5 [18]. The anticatabolic effects of factors, such as caspase inhibitor [19], tissue inhibitor of metalloproteinase-1 [20], IL-1 receptor, anti-TNF- $\alpha$ antagonists [12,21,22], and others have been shown on the extracellular matrix metabolism of IVD cells in vitro.

The anticatabolic role of the specific inhibition of ADAMTS4 and ADAMTS5 in human cartilage was recently found by using a small interfering RNA (siRNA) approach in normal and osteoarthritic explants [23]. To date, no report has been made that an anticatabolic factor suppresses IVD degeneration in vivo. The purpose of this study was to prove our hypothesis that a single injection of ADAMTS5 siRNA inhibits the production of ADAMTS5 and suppressed IVD degeneration in the rabbit anular needle-puncture model.

\section{Materials and methods In vitro study}

Cell preparation and alginate bead culture

Lumbar IVDs from four consecutive levels (L2/3, L3/4, L4/5, and $L 5 / S 1)$ were dissected from the spines of adolescent Japanese white rabbits ( 1 to $1.5 \mathrm{~kg}, 6$ to 9 weeks old) after killing by injection of an excess amount of sodium pentobarbital (Dainippon Pharmaceutical, Osaka, Japan). Tissues were separately harvested from the NP and the outer layer of the AF, and the cells were isolated by using a sequential proteinase and collagenase digestion, as previously described [24]. Primary cells were expanded in monolayer culture in complete media (Dulbecco's Modified Eagles Medium (DMEM) supplemented with $10 \% \mathrm{FBS}, 100 \mathrm{U} / \mathrm{ml}$ penicillin, $100 \mu \mathrm{g} / \mathrm{ml}$ streptomycin, and $0.5 \mu \mathrm{g} / \mathrm{ml}$ amphotericin B (Fungizone)). After one passage, cells were suspended in sodium alginate $(1.2 \%$ solution in $155 \mathrm{mM} \mathrm{NaCl}$; Cambrex CC-3234, Charles, lowa, USA) at a density of $5 \times 10^{5}$ cells $/ \mathrm{ml}$. The beads were maintained for up to 14 days with Chondrocyte Differentiation Media (Cambrex CC-3225) and seeded in a 12-well plate at a density of $1 \times 10^{5}$ cells/well.

\section{Establishment of siRNA for ADAMTS5 oligonucleotide and transient transfection}

The siRNA oligonucleotide for the rabbit ADAMTS5 gene was constructed from a completely homologous region of sequences in the ADAMTS5 gene of the human, rat, and mouse from the NCBI website [25]. The reverse transcriptasepolymerase chain reaction (RT-PCR) primers were constructed from this homologous region, and RT-PCR was completed. PCR products were collected, and the rabbit ADAMTS5 gene was cloned by using the TA Cloning Kit (Invitrogen, Carlsbad, CA, USA) and confirmed by sequencing by using the ABI PRISM 310. Sequences for primers used in 
these analyses were as follows: 5'-CTCCCAGGACAAACCTACGA-3' and 5'-CCTCTTCCCTGTG CAGTAGC-3' for ADAMTS 5 cDNA amplification. SiRNA for the ADAMTS5 oligonucleotide was constructed by using the Takara Website [26]. Sequences for the control and ADAMTS5 oligonucleotides of the siRNA used in these analyses were as follows: sense oligonucleotide 5'-CGAUCCUCAAAGCACUACUTT3', anti-sense oligonucleotide 5'-AGUA GUGCUUUGAGGAUCGTT-3' for the control, sense oligonucleotide 5'-CCACCAUCACG GAAUUCCUTT-3', and anti-sense oligonucleotide 5'-AGGAAUUCCGUGAUGGUGGTT-3' for ADAMTS5. Sense and antisense oligonucleotide siRNAs were separately dimerized for the control and ADAMTS5.

\section{Confirmation of the knockdown rate of the ADAMTS5 GENE with or without IL-1 $\beta$ stimulation in monolayer culture}

A standard for the rabbit ADAMTS5 gene was constructed from the sequence originally analyzed. For transient transfection, NP cells were seeded in a 12-well plate at a density of 1 $\times 10^{5} \mathrm{cells} /$ well and cultured in complete media.

\section{Effect on constitutive expression}

After 48 hours, the NP cells were transiently transfected with the anti-ADAMTS5 oligonucleotide or control oligonucleotide, added directly to media without transfection reagents. After 48 hours' incubation, the cells were harvested, and the mRNA level of ADAMTS5 was assessed.

\section{Effect on IL-1 $\beta$-stimulated expression}

After 48-hour preculture, cells were cultured with or without IL$1 \beta(10 \mathrm{ng} / \mathrm{ml})$ (Roche, Mannheim, Germany) in serum-free DMEM. After 24 hours of IL-1 treatment, anti-ADAMTS5 oligonucleotide or control oligonucleotide was added to the culture for transfection. After 24 hours' incubation with siRNA, NP cells were collected and subjected to mRNA analysis.

\section{Confirmation of the knockdown rate of the ADAMTS5 gene by "siSTABLE" siRNA in alginate bead culture} Freshly prepared stable anti-ADAMTS5 oligonucleotide (Dharmacon siSTABLE, Thermo Scientific, Lafayette, CA, USA) was prepared for in vivo experiments, but first tested in an in vitro alginate culture system. NP cells were cultured in alginate beads, as described earlier. After 14 days, NP cells in alginate beads were transfected with anti-ADAMTS5 oligonucleotide or control oligonucleotide (Dharmacon) without transfection reagents. Results are reported normalized to GAPDH.

\section{RNA isolation and real-time PCR}

Total RNA was extracted from transfected cells by using Isogen (Nippongene, Tokyo, Japan) and purified with the SV Total RNA Isolation System (Promega, Madison, WI, USA).

Random-primed cDNAs were synthesized by using Multiscribe reverse transcriptase (PE Applied Biosystems, Foster,
CA, USA). Quantitative real-time PCR was carried out by using a PRISM 7700 sequence detector with the QuantiTect SYBR Green PCR kit (Qiagen, Valencia, CA, USA) according to the manufacturer's instructions. The relative expression of ADAMTS5 was calculated by using the comparative threshold $\left(\mathrm{C}_{\mathrm{T}}\right)$ method, as previously described [27]. Results are reported normalized to the housekeeping gene glyceraldehyde 3-phosphate dehydrogenase (GAPDH).

\section{In vivo study}

Establishment of a degenerative IVD by using the rabbit anular needle-puncture model and injection of the antiADAMTS5 oligonucleotide

An anular puncture model was established by using an 18gauge needle at a defined depth of puncture $(5 \mathrm{~mm})$, as previously reported [28]. New Zealand white rabbits $(n=12)$, weighing approximately 3.5 to $4.0 \mathrm{~kg}$ (5 months old), were used in this study with the approval of the Institutional Animal Care and Use Committee (06-067). Under general anesthesia, lumbar IVDs were exposed, and the initial puncture with an 18-gauge needle was performed on two noncontiguous discs ( $L 2 / 3$ and $L 4 / 5)$, with the disc (L3/4) between the punctured discs left intact as a control [17]. One week after the initial puncture, the discs were exposed again from the contralateral side, and either control siRNA or ADAMTS5 siRNA oligonucleotide (Dharmacon, was injected into the center of the NP by using a 26 -gauge needle $(10 \mu \mathrm{g}$ in $10 \mu \mathrm{l}$ phosphate-buffered saline (PBS) per disc). The timing of injection was earlier than that used in other studies $[17,18]$ to reveal the effect of an injection of ADAMTS5 siRNA during the acute phase of disc degeneration. Nine weeks after the initial anular puncture (8 weeks after the injection), all rabbits were killed.

\section{Radiographic analysis of disc height}

Radiographs were taken at time 0 and at weeks 1, 2, 3, 5, 7, and 9 after the puncture. Extreme care was taken to maintain a consistent level of anesthesia during radiography of each animal at each time point to obtain a similar degree of muscle relaxation, which may affect the disc height. Therefore, the preoperative radiograph was always used as a baseline measurement. Radiographs were digitally scanned and digitally stored by using an image-capture software program.

\section{Image analysis}

All radiographic images were independently analyzed by using a custom program for MATLAB software (Natick, MA, USA) by an orthopedic researcher who was blinded to the treatment groups. Data are reported as the IVD height expressed as the disc-height index (DHI) (DHI = intervertebral disc height/adjacent vertebral body height) [17]. Changes in the disc-height index of injected discs were expressed as percentage $\mathrm{DHI}$ $(\% \mathrm{DHI})$ and normalized to the measured preoperative intervertebral disc height $(\% \mathrm{DHI}=($ Postoperative DHI/Preoperative $\mathrm{DHI} \times 100)$ [17]. To avoid the influence of anesthesia, the $\% \mathrm{DHI}$ at the experimental level was further normalized to 
$\% \mathrm{DHI}$ at the nonpunctured level (normalized \%DHI = (Punctured \%DHI/Nonpunctured \%DHI) $\times 100$ ).

\section{MRl assessment}

MRI examinations were performed on all rabbits in the study by using a 0.3-T imager (Airis II, version 4.0 A; Hitachi Medical System America, Inc., Twinsburg, Ohio, USA) with a quadrature extremity coil receiver. After killing, the spinal columns with surrounding soft tissue were isolated and subjected to MRI analysis [17]. An observer, blinded to the study groups, used a modified Thompson classification based on changes in the degree and area of signal intensity from grade 1 to 4 (1= normal, 2 = minimal decrease in signal intensity but obvious narrowing of high-signal area, $3=$ moderate decrease in signal intensity, and $4=$ severe decrease in signal intensity) to evaluate the MRIs. The intraobserver and interobserver reliability correlation coefficients of MRI grading based on two evaluations were excellent ( $K=0.98,0.90$, respectively), as determined by the Cohen kappa correlation coefficient [28].

\section{Histologic evaluations}

After MRI assessment, the experimental IVDs were excised from the vertebral body-disc-vertebral body unit, and each IVD was fixed in 10\% formalin, decalcified, embedded in paraffin, sectioned, and assessed with conventional histology and immunostaining. Midsagittal sections $(5 \mu \mathrm{m})$ of each IVD were stained either with hematoxylin and eosin or with safranin-O. An observer, blinded to the experiment, analyzed the histologic sections and graded them by using our recently established protocol [28].

\section{Statistics}

The \%DHI was statistically analyzed by using a two-way repeated analysis of variance and Fisher protected least significant difference as a post hoc test (factors; time and treatment group). Other statistical analyses were assessed with the Mann-Whitney $U$ test. In vitro experiments were performed 3 times.

\section{Results In vitro study}

Rabbit NP cells were chosen because their response to IL-1 $\beta$ was more consistent than that of AF cells (as determined from previous experiments) and because the injection site in the NP has a higher concentration of proteoglycans. The effectiveness of administration of ADAMTS5 siRNA might be influenced by the rich positively charged matrix in NP tissues.

Effect of siRNA on rabbit ADAMTS5 gene expression in rabbit NP cells cultured in monolayer with or without stimulation with IL-1B

We confirmed that the siRNA oligonucleotide we constructed knocked down the ADAMTS5 gene in rabbit NP cells. At 48 hours after transfection, the NP cells that received the ADAMTS5 siRNA oligonucleotide showed approximately a
Figure 1

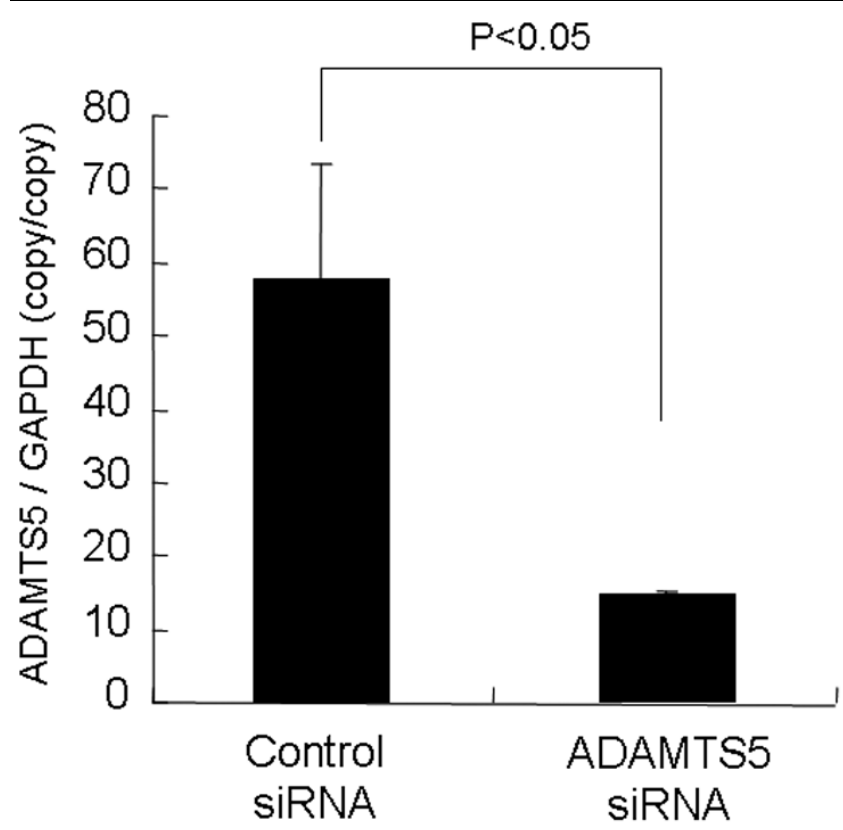

Establishment of small interfering RNA (siRNA) oligonucleotide for ADAMTS5 in rabbit nucleus pulposus (NP) cells. After the 48-hour preculture period, rabbit NP cells were transfected with the siRNA oligonucleotide specific for either the control or ADAMTS5. At 48 hours after transfection in NP cells, the ADAMTS5 siRNA-transfected cells showed approximately a 75\% knock-down of ADAMTS5 mRNA compared with the control siRNA. The results are reported normalized to glyceraldehyde 3-phosphate dehydrogenase (GAPDH).

$75 \%$ knockdown of constitutive expression of ADAMTS5 mRNA (Figure 1). This suppression was observed in all three experiments. Real-time PCR revealed that IL-1 $\beta$ treatment for 24 hours increased the abundance of mRNA for ADAMTS5 (about 12-fold) in a dose-dependent manner in rabbit NP cells (Figure 2a). Based on these results, a concentration of IL-1 $\beta$ of $10 \mathrm{ng} / \mathrm{ml}$ was chosen for further studies. Subsequently, after IL- $1 \beta$ treatment at $10 \mathrm{ng} / \mathrm{ml}$ for 24 hours, NP cells were transfected with ADAMTS5 and control siRNA. At 24 hours after the transfection, the abundance of ADAMTS5 mRNA was knocked down by $70 \%$ compared with the control group in rabbit NP cells (Figure 2b). This effect persisted for 2 weeks (data not shown).

\section{Effect of adamts5 oligonucleotide on rabbit NP cells cultured in alginate beads}

It is possible that the injected siRNA cannot penetrate the matrix of the target tissue and induce metabolic changes. To confirm our hypothesis that ADAMTS5 siRNA has an effect in a three-dimensional environment, the efficacy of ADAMTS5 was tested by using the alginate bead-culture system. We confirmed that the ADAMTS5 gene was significantly knocked down by using $A D A M T S 5$ siRNA with no transfection reagent. The knockdown rate of the ADAMTS5 siRNA cells was $70 \%$ 

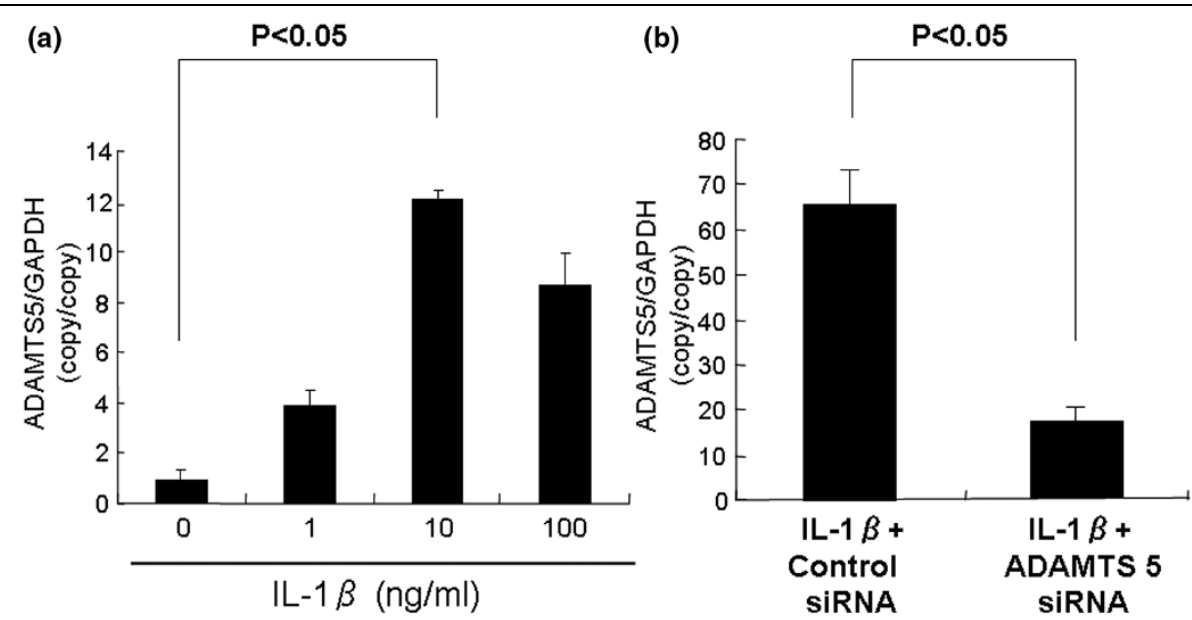

Effect of interleukin-1 $\beta$ (IL-1 $\beta$ ) stimulation on ADAMTS5 mRNA expression in rabbit nucleus pulposus (NP) cells. After real-time polymerase chain reaction (PCR), the ADAMTS5 mRNA expression level after IL-1 $\beta$ stimulation (24 hours) in rabbit NP cells is shown (a). IL-1 $\beta$ at $10 \mathrm{ng} / \mathrm{ml}$ induced the highest level of increased expression of mRNA for ADAMTS5 (about 12-fold); that concentration was chosen for subsequent studies. (b) NP cells seeded in a 12-well plate at a density of $1 \times 10^{5} \mathrm{cells} /$ well. After the 48-hour preculture period, cells were cultured in serum-free media in the presence of IL-1 $\beta(10 \mathrm{ng} / \mathrm{ml})$ for 24 hours. After the 24-hour treatment with IL-1 $\beta$, NP cells were transiently transfected with the anti-ADAMTS5 oligonucleotide or control oligonucleotide by adding oligonucleotide directly to the culture media. Twenty-four hours later, NP cells were collected, and the expression of ADAMTS5 was analyzed with real-time PCR. ADAMTS5 mRNA expression was knocked down by about $70 \%$ in rabbit NP cells that were transfected with ADAMTS5 siRNA and stimulated with IL-1 $\beta(10 \mathrm{ng} / \mathrm{ml})$. The results are reported normalized to GAPDH.

Figure 3

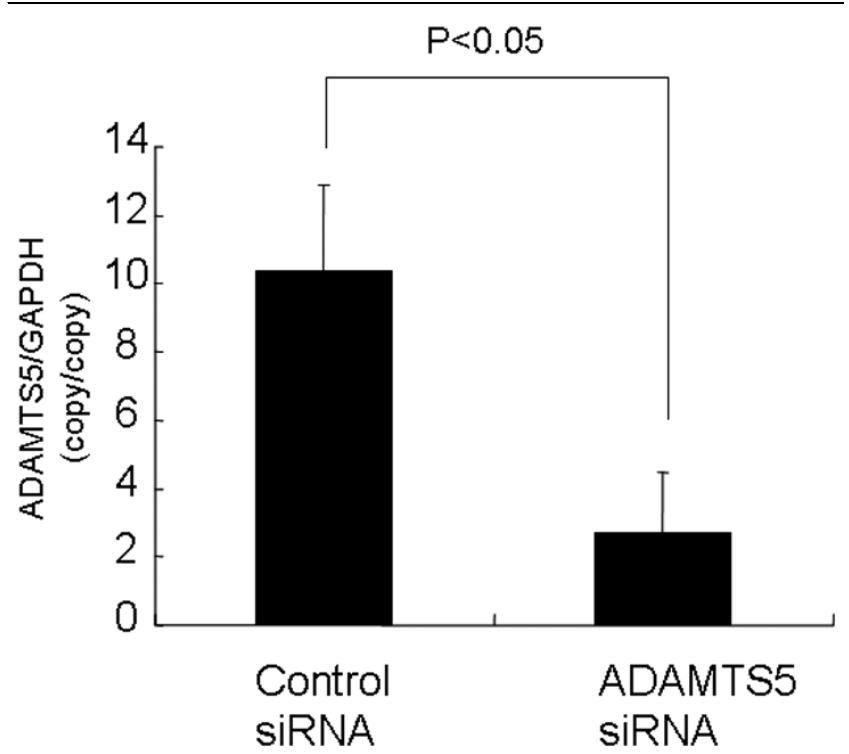

Effect of ADAMTS5 oligonucleotide on rabbit nucleus pulposus (NP) cells in alginate bead culture. NP cells were suspended in sodium alginate at a density of $5 \times 10^{5} \mathrm{cells} / \mathrm{ml}$ and maintained for up to 14 days. After 14 days, the NP cells in alginate beads were transfected with antiADAMTS5 oligonucleotide or control oligonucleotide without using gene-delivery reagents. In NP cells in alginate bead culture, the expression of the ADAMTS5 gene was approximately $70 \%$ of that seen with the control oligonucleotide. The results are reported normalized to GAPDH.
(Figure 3); this effect also persisted for about 2 weeks (data not shown).

\section{In vivo Study}

\section{Radiographic assessment}

Radiographic assessments were performed to confirm that the rabbits received identical punctures with an 18-gauge needle at the correct levels. The needle puncture was found to have reduced the $\% \mathrm{DHI}$ at 1 week $(70 \% \mathrm{DHI} ; P>0.06$, Figure 4$)$, with no significant difference in $\% \mathrm{DHI}$ between the two groups. At that time, the control oligonucleotide or antiADAMTS5 oligonucleotide (100 $\mu \mathrm{g}$ in $10 \mu \mathrm{l}$ of PBS) was administered into the NP of the rabbit IVDs. After the siRNA injections, no significant differences were found in the $\% \mathrm{DHI}$ between the control siRNA and the ADAMTS5 siRNA groups during the observation period (Figure 4).

\section{$M R I$ analysis}

MRI analysis was performed at 8 weeks after the siRNA injections when the animals were killed. The MRI of the NP in the ADAMTS5 siRNA group showed a stronger $\mathrm{T}_{2}$ signal intensity than that found in the Control group (Figure 5). When disc degeneration was assessed by using the Thompson MRI grading score, the grading score was significantly lower (better) in the ADAMTS5 siRNA group than in the control siRNA group $(P=0.02$, Mann-Whitney $U$ test) (Figure 6). 
Figure 4

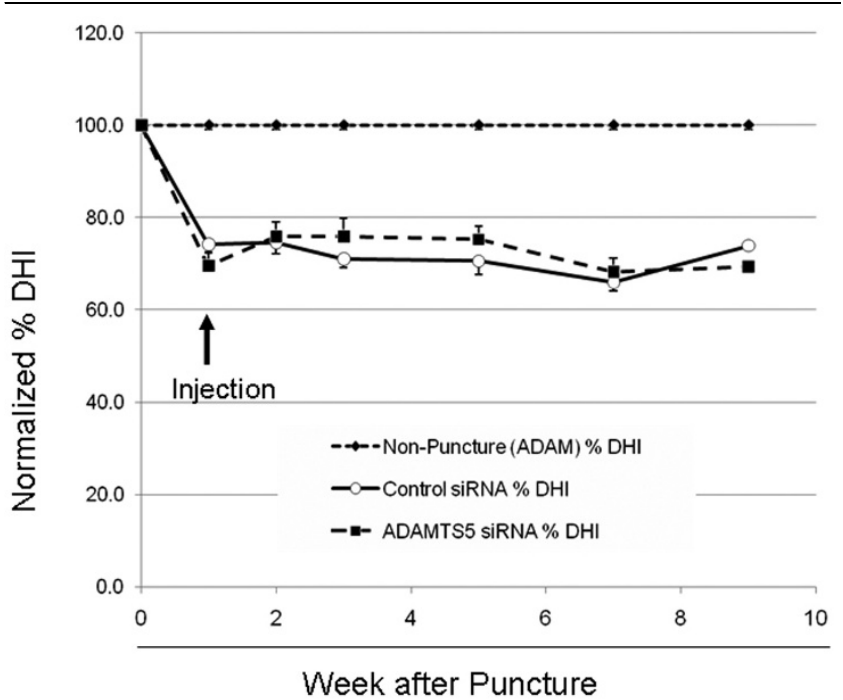

Radiographic assessment in the rabbit anular puncture model of disc degeneration. An anular puncture model was established in 5-monthold New Zealand white rabbits. Under general anesthesia, lumbar intervertebral discs were exposed, and the initial puncture with an 18gauge needle at a defined depth of puncture $(5 \mathrm{~mm})$ was performed on two noncontiguous discs (L2/3 and L4/5), with the disc (L3/4) between the punctured discs left intact as a control. One week after the initial puncture, either control small interfering RNA (siRNA) or ADAMTS5 siRNA oligonucleotide $(10 \mu \mathrm{g}$ in $10-\mu \mathrm{l}$ phosphate-buffered saline (PBS) per disc) was injected into the center of the nucleus pulposus by using a 26 -gauge needle. Nine weeks after the initial anular puncture ( 8 weeks after the injection), all rabbits were killed. Radiographs were taken at time 0 and at weeks $1,2,3,5,7$, and 9 after the puncture to quantity changes in the disc-height index (DHI). The \%DHI was calculated as $[\% \mathrm{DHI}=($ Postoperative $\mathrm{DH} /$ Preoperative $\mathrm{DHI}) \times$ 100]. At 8 weeks after the ADAMTS5 siRNA injection, no difference was found in the mean \%DHI of the ADAMTS5 siRNA-injected punctured discs compared with the punctured discs that received the control siRNA injection ( $P>0.05$, repeated ANOVA).

\section{Histologic evaluation}

The injection of ADAMTS5 siRNA significantly affected the histochemical changes found with IVD degeneration. Eight weeks after the control or ADAMTS5 siRNA injections, the control siRNA group displayed a complete loss of NP tissues, which had been replaced by a fibrocartilaginous tissue (Figure $7 \mathrm{a}$ and $7 \mathrm{c}$ ). The severely degenerated discs that had received the control siRNA showed a loss of proteoglycans and the collapsed, wavy fibrocartilage lamellae typical of the AF with associated fibrochondrocytes (Figure $7 \mathrm{e}$ and $7 \mathrm{~g}$ ). In the discs that received the ADAMTS5 siRNA, safranin-O staining demonstrated the maintenance of IVD structure with a lightly stained matrix and large cells (Figure $7 \mathrm{~b}$ and $7 \mathrm{~d}$ ); the NP was rounded and well filled with numerous large, vacuolated cells and smaller chondrocyte-like cells typical of the normal IVD (Figure $7 \mathrm{f}$ and $7 \mathrm{~h}$ ). The histologic grading scores demonstrate that the cellularity and matrix of the NP in the ADAMTS5 siRNA-treated discs were significantly lower (better) than those in the control siRNA group (Figure 8). The total grading
Figure 5

$\mathrm{L} 2 / 3$

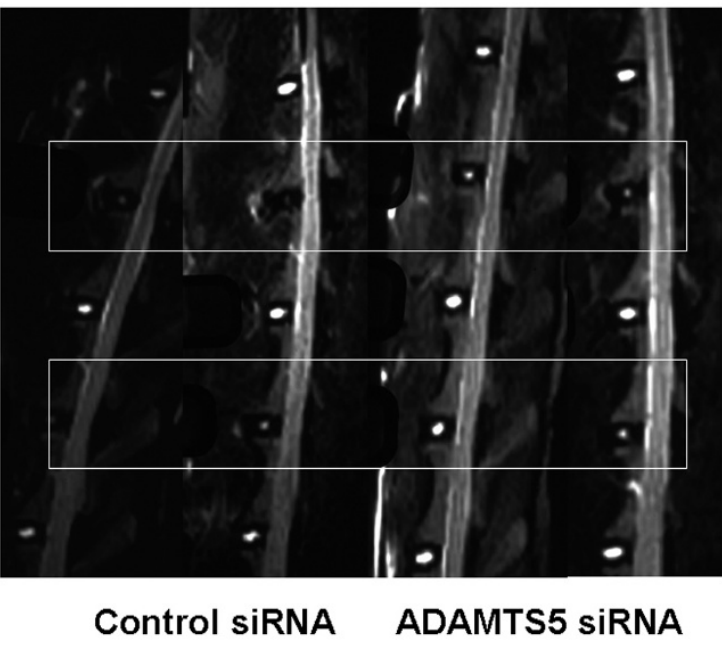

Magnetic resonance imaging (MRI) findings after small interfering RNA (siRNA) oligonucleotide injection in the rabbit anular puncture model of disc degeneration. MRI examinations were performed on all spinal columns isolated from the rabbits ex vivo at death 8 weeks after the siRNA oligonucleotide injection. In these representative MRIs, the $\mathrm{T}_{2}$ signal intensity in the nucleus pulposus of the ADAMTS5 siRNA-injected discs was stronger than that in the control siRNA-injected discs.

score (SUM) in the ADAMTS5 siRNA-treated discs was also significantly lower than those in the control siRNA group (Mann-Whitney; $P<0.05$ ) (Figure 8). The scores for the AF and the border between the AF and NP in the ADAMTS5 siRNA-treated discs showed a trend to be lower than those in the control discs (Mann-Whitney; AF; $P=0.06$; border between the AF and NP; $P=0.08$ ).

\section{Discussion}

With the rabbit anular puncture model, this study explored the efficacy of a direct injection of ADAMTS5 siRNA into the NP on the delay or attenuation of disc degeneration. Our results demonstrate that the designed ADAMTS5 siRNA was (a) active in vitro and (b) effective in suppressing the degeneration of the NP tissue in the in vivo rabbit model. However, the injection of ADAMTS5 siRNA did not induce the anticipated recovery of disc height.

We successfully designed and constructed an siRNA oligonucleotide with biologic activity for the rabbit ADAMTS5 gene. ADAMTS5 siRNA-transfected rabbit NP cells showed approximately a $75 \%$ knockdown of ADAMTS5 mRNA compared with the control siRNA. Although we demonstrated that IL-1 $\beta$ treatment significantly increased the ADAMTS 5 mRNA level in NP cells, the suppression of the expression of the ADAMTS5 gene by ADAMTS5 siRNA was $70 \%$ compared with the control oligonucleotide in both monolayer and alginate bead culture under stimulation with IL-1 $\beta$. 


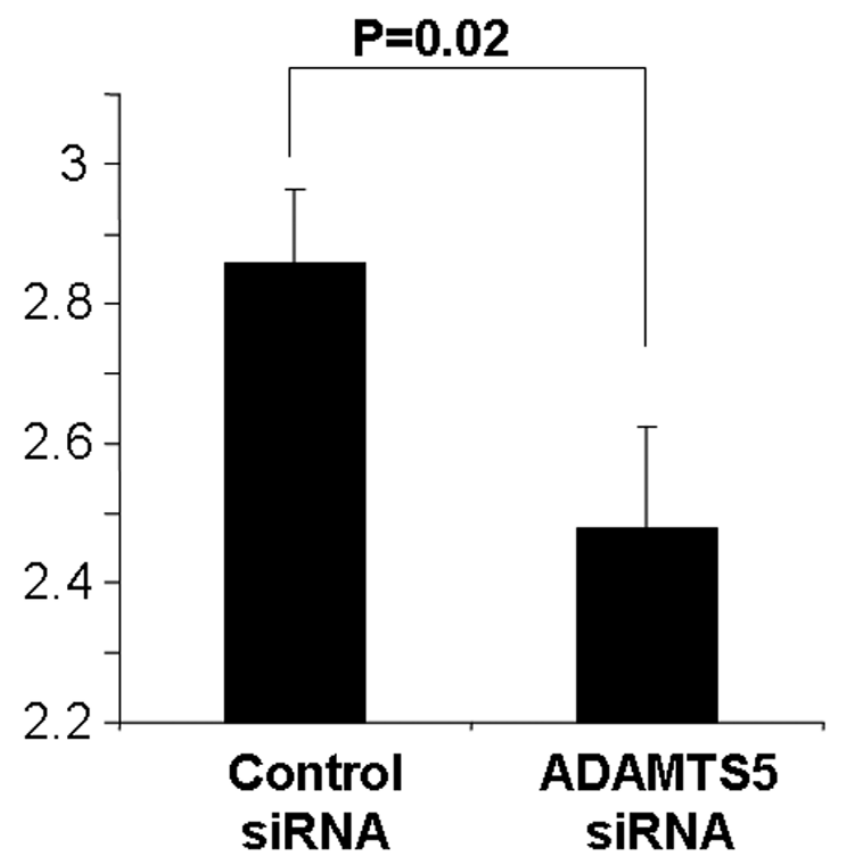

Magnetic resonance imaging (MRI) assessment 8 weeks after small interfering RNA (siRNA) injection in the rabbit anular puncture model of disc degeneration. An observer blinded to the study assessed the MRIs by using the modified Thompson scale, based on changes in the degree and area of signal intensity from grades 1 to 4 . After assessment of the MRI grades, a significantly lower (better) MRI grade in the ADAMTS5 siRNA-injected discs was observed compared with the control siRNA-injected discs ( $P=0.02$, Mann-Whitney test).

The intradiscal injection of ADAMTS5 siRNA during the acute phase of disc degeneration after anular puncture in the rabbit delayed the progression of disc degeneration, as assessed by MRI scores, signal intensity of NP on MRI, and histologic scores. MRI findings of a high $\mathrm{T}_{2}$ signal intensity in the NP indicate that the NP in the punctured discs treated with ADAMTS5 siRNA was hydrated. Therefore, ADAMTS5, which cleaves the core protein of aggrecan, may significantly contribute to the loss of water content of the NP after anular puncture. However, the reason that the disc height loss was not reversed by the injection of ADAMTS siRNA remains to be determined. One possible explanation is that the treatment with siRNA for ADAMTS5 is an anticatabolic one, not anabolic. In addition, a possibility remains that the injected siRNA was retained in the NP area, where the siRNA was injected, by some mechanism, or mainly internalized by NP cells and did not distribute to the AF area. In a previous study using the rabbit anular puncture model, the injection of osteogenic protein1 into the NP induced an increased proteoglycan content of both the AF and the NP and the recovery of disc-height loss by 6 weeks [17]. One could speculate that the maintenance of disc height is determined by the structural integrity of the anulus, which could not be fully assessed through MRI and histology in a quantitative fashion. Furthermore, we did not test
Figure 7

\section{Control siRNA \\ ADAMTS5 SIRNA}
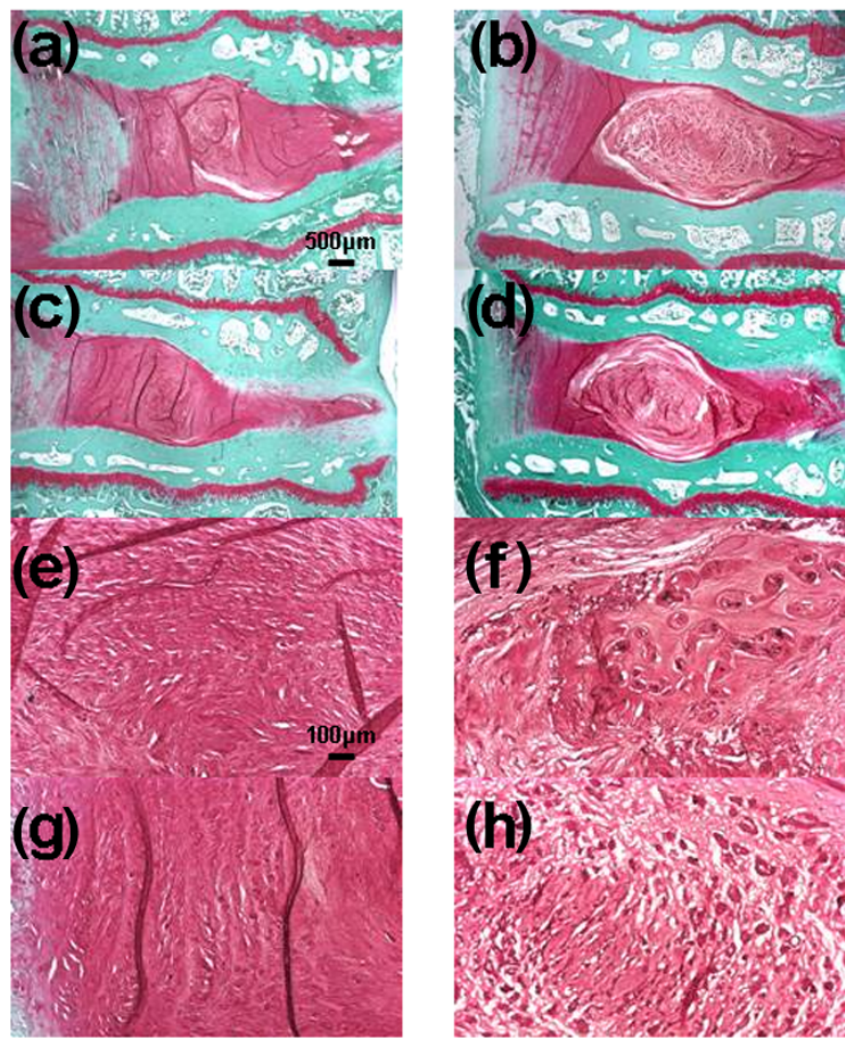

Safranin-O-stained sections reflecting typical histologic changes after injection of control small interfering RNA (siRNA) or ADAMTS5 siRNA in the rabbit anular puncture model of disc degeneration. Eight weeks after the control or ADAMTS5 siRNA injections, the control siRNA group displayed a complete loss of nucleus pulposus (NP) tissues, which had been replaced by a fibrocartilaginous tissue $(a, c)$. The severely degenerated discs that had received the control siRNA showed a loss of proteoglycans and the collapsed, wavy fibrocartilage lamellae typical of the anulus fibrosus (AF), with associated fibrochondrocytes $(\mathbf{e}, \mathbf{g})$. In the ADAMTS5 siRNA-injected discs, safranin-O staining demonstrated the maintenance of intervertebral disc structure with a lightly stained matrix and large cells $(b, d)$; the NP was rounded and bloated looking, and consisted of numerous large, vacuolated cells and smaller chondrocyte-like cells $(\mathbf{f}, \mathbf{h})$. A clear demarcation was seen between the NP and inner anulus in the ADAMTS5 siRNA-injected discs. (Magnification $\times 20$ (a-d), $\times 100(e-h)$ ). The level in $a, b, e$, and $f$ is $\mathrm{L} 2 / 3$, and in $\mathrm{c}, \mathrm{d}, \mathrm{g}$, and $\mathrm{h}$ is $\mathrm{L} 4 / 5$.

different doses of ADAMTS5 siRNA in the anular puncture model, nor did we assess the half-life of injected siRNA. The limited effects of ADAMTS5 siRNA may point to a complex involvement of multiple enzymes in disc degeneration. Nevertheless, the strong suppression of the ADAMTS5 gene by siRNA in vitro and in vivo, especially in NP tissues, indicates that ADAMTS5 might play an important role in IVD degeneration.

Histologic findings from safranin-O staining were supportive of the maintenance of NP tissues in the ADAMTS5 siRNA- 

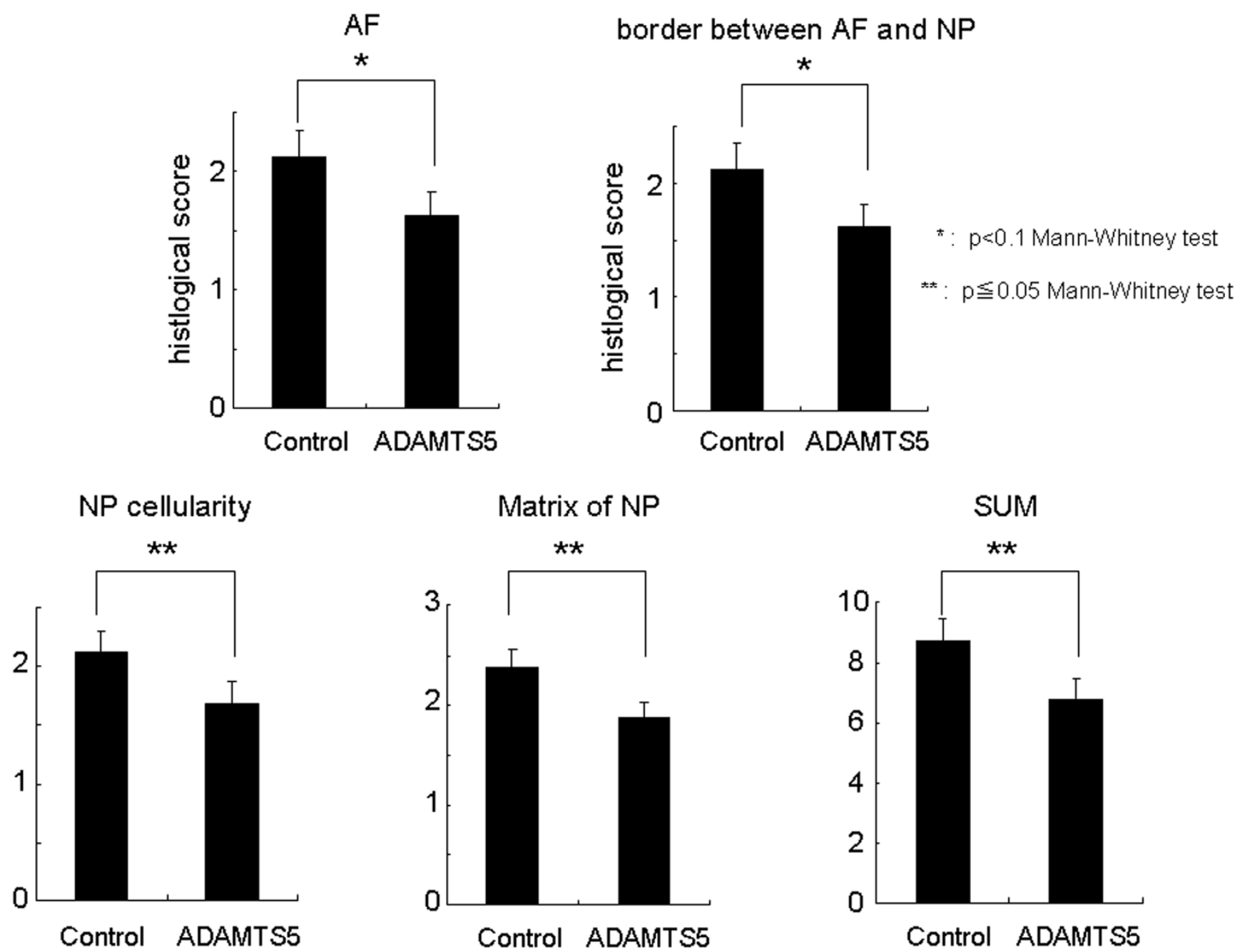

Histologic assessment after ADAMTS5 small interfering RNA (siRNA) or control siRNA injection in the rabbit anular puncture model of disc degeneration. In ADAMTS5 siRNA-injected discs, the anulus fibrosus (AF) and the border between the AF and nucleus pulposus (NP) showed a tendency to have a lower (better) histologic score than the control siRNA-injected discs (Mann-Whitney test; $P<0.1$ ). In the NP, the cellularity, matrix, and total grading score (SUM) were significantly better in the ADAMTS5 siRNA-injected discs than in the control siRNA-injected discs (Mann-Whitney test; $P<0.05)$.

treated discs, as observed with MRI. The improving histologic scores associated with the NP might indicate that ADAMTS5 is more involved in matrix degeneration of the NP than that in the AF. In addition, the direct injection of siRNA into the NP may induce a localized improvement. It is worth noting that the histologic scores for the AF and the border between the AF and NP in the ADAMTS5 siRNA-treated discs showed a trend to be lower (improved) when compared with those for the control discs. These findings might indicate that the inhibition of degeneration or improved reparative activity of the NP may have contributed to the improved histologic grading for the AF and the border between the AF and NP.

The treatment of human disc cells with IL-1 induced an imbalance between catabolic and anabolic events, responses that represent the changes seen during disc degeneration $[12,13,29]$. After treatment with IL-1, the aggrecanases (ADAMTS4, 5), matrix metalloproteinase-3 (MMP-3), and $M M P-13$, gene expression was increased in cells derived from the human NP cells [13]. Séguin and colleagues [30] reported that induction of ADAMTS4 and -5 mRNA occurred downstream of NF- $\kappa \beta$ activation in NP cells. These results, and the recent reports on the contribution of IL-1 in disc degeneration [31], may indicate that as disc degeneration progresses, more ADAMTS5 is expressed in the IVD, with a high association with an increased amount of IL-1.

In summary, we have shown evidence that the suppression of ADAMTS5 in turn suppressed IVD degeneration; this suggests the possible contribution of ADAMTS5 to disc degener- 
ation, especially in the NP of the rabbit anular puncture model of disc degeneration.

\section{Conclusions}

A single injection of ADAMTS5 siRNA suppressed disc degeneration in the NP, as shown by the significantly improved MRI and histologic grades. The results may suggest that ADAMTS5 contributes to the degeneration of NP tissues in the rabbit anular puncture disc-degeneration model. The mechanism for the differences in response to siRNA in disc height and MRI findings may be worthy of exploration.

\section{Competing interests}

The authors declare that they have no competing interests.

\section{Authors' contributions}

SS conceived this study and made substantial contributions to the study design and to writing the manuscript. SS also acquired and interpreted the data. YA and KA performed data acquisition, statistical analysis, and interpretation of data. KM participated in the design of the study and finalized the manuscript. YK and TK participated in the design of the study and performed the statistical analysis. CM performed the histologic analyses. Al carried out the in vitro assay of ADAMTS5 siRNA.

\section{Acknowledgements}

We thank Ms. Mary Ellen Lenz for her assistance in the preparation of the manuscript. This work and her assistance was supported in part by a Grant-in-Aid for young scientists (A) 18689903 (SS), and NIH grants 1-P01-AR48152 (KM).

\section{References}

1. Sandy JD, Flannery CR, Neame PJ, Lohmander LS: The structure of aggrecan fragments in human synovial fluid: evidence for the involvement in osteoarthritis of a novel proteinase which cleaves the Glu 373-Ala 374 bond of the interglobular domain. J Clin Invest 1992, 89:1512-1516.

2. Lohmander LS, Neame PJ, Sandy JD: The structure of aggrecan fragments in human synovial fluid: evidence that aggrecanase mediates cartilage degradation in inflammatory joint disease, joint injury, and osteoarthritis. Arthritis Rheum 1993, 36:1214-1222.

3. Kuno K, Okada Y, Kawashima H, Nakamura H, Miyasaka M, Ohno $\mathrm{H}$, Matsushima $\mathrm{K}$ : ADAMTS-1 cleaves a cartilage proteoglycan, aggrecan. FEBS Lett 2000, 478:241-245.

4. Rodriguez-Manzaneque JC, Westling J, Thai SN, Luque A, Knauper V, Murphy G, Sandy JD, Iruela-Arispe ML: ADAMTS1 cleaves aggrecan at multiple sites and is differentially inhibited by metalloproteinase inhibitors. Biochem Biophys Res Commun 2002, 293:501-508.

5. Somerville RP, Longpre JM, Jungers KA, Engle JM, Ross M, Evanko S, Wight TN, Leduc R, Apte SS: Characterization of ADAMTS-9 and ADAMTS-20 as a distinct ADAMTS subfamily related to Caenorhabditis elegans GON-1. J Biol Chem 2003, 278:9503-9513.

6. Collins-Racie LA, Flannery CR, Zeng W, Corcoran C, Annis-Freeman B, Agostino MJ, Arai M, DiBlasio-Smith E, Dorner AJ, Georgiadis KE, Jin $M$, Tan XY, Morris EA, LaVallie ER: ADAMTS-8 exhibits aggrecanase activity and is expressed in human articular cartilage. Matrix Biol 2004, 23:219-230.

7. Glasson SS, Askew R, Sheppard B, Carito B, Blanchet T, Ma HL, Flannery CR, Peluso D, Kanki K, Yang Z, Majumdar MK, Morris EA: Deletion of active ADAMTS5 prevents cartilage degradation in a murine model of osteoarthritis. Nature 2005, 434:644-648.
8. Glasson SS, Askew R, Sheppard B, Carito BA, Blanchet T, Ma HL, Flannery CR, Kanki K, Wang E, Peluso D, Yang Z, Majumdar MK, Morris EA: Characterization of and osteoarthritis susceptibility in ADAMTS-4-knockout mice. Arthritis Rheum 2004, 50:2547-2558.

9. Majumdar MK, Askew R, Schelling S, Stedman N, Blanchet T, Hopkins B, Morris EA, Glasson SS: Double-knockout of ADAMTS-4 and ADAMTS-5 in mice results in physiologically normal animals and prevents the progression of osteoarthritis. Arthritis Rheum 2007, 56:3670-3674.

10. Sztrolovics R, Alini M, Roughley PJ, Mort JS: Aggrecan degradation in human intervertebral disc and articular cartilage. Biochem J 1997, 326(Pt 1):235-241.

11. Patel KP, Sandy JD, Akeda K, Miyamoto K, Chujo T, An HS, Masuda K: Aggrecanases and aggrecanase-generated fragments in the human intervertebral disc at early and advanced stages of disc degeneration. Spine 2007, 32:2596-2603.

12. Le Maitre CL, Freemont AJ, Hoyland JA: A preliminary in vitro study into the use of IL-1Ra gene therapy for the inhibition of intervertebral disc degeneration. Int J Exp Pathol 2006, 87:17-28.

13. Le Maitre CL, Freemont AJ, Hoyland JA: The role of interleukin-1 in the pathogenesis of human intervertebral disc degeneration. Arthritis Res Ther 2005, 7:R732-R745.

14. Patwari P, Gao G, Lee JH, Grodzinsky AJ, Sandy JD: Analysis of ADAMTS4 and MT4-MMP indicates that both are involved in aggrecanolysis in interleukin-1-treated bovine cartilage. Osteoarthritis Cartilage 2005, 13:269-277.

15. Pattoli MA, MacMaster JF, Gregor KR, Burke JR: Collagen and aggrecan degradation is blocked in interleukin-1-treated cartilage explants by an inhibitor of IkappaB kinase through suppression of metalloproteinase expression. J Pharmacol Exp Ther 2005, 315:382-388.

16. Bau B, Gebhard PM, Haag J, Knorr T, Bartnik E, Aigner T: Relative messenger RNA expression profiling of collagenases and aggrecanases in human articular chondrocytes in vivo and in vitro. Arthritis Rheum 2002, 46:2648-2657.

17. Masuda K, Imai $Y$, Okuma M, Muehleman $C$, Nakagawa $K$, Akeda K, Thonar E, Andersson G, An HS: Osteogenic protein-1 injection into a degenerated disc induces the restoration of disc height and structural changes in the rabbit anular puncture model. Spine 2006, 31:742-754.

18. Chujo T, An HS, Akeda K, Miyamoto K, Muehleman C, Attawia M, Andersson G, Masuda K: Effects of growth differentiation factor- 5 on the intervertebral disc: in vitro bovine study and in vivo rabbit disc degeneration model study. Spine 2006, 31:2909-2917.

19. Park JB, Park IC, Park SJ, Jin HO, Lee JK, Riew KD: Anti-apoptotic effects of caspase inhibitors on rat intervertebral disc cells. $J$ Bone Joint Surg Am 2006, 88:771-779.

20. Wallach CJ, Sobajima S, Watanabe Y, Kim JS, Georgescu HI, Robbins P, Gilbertson LG, Kang JD: Gene transfer of the catabolic inhibitor TIMP-1 increases measured proteoglycans in cells from degenerated human intervertebral discs. Spine 2003, 28:2331-2337.

21. Hoyland JA, Le Maitre C, Freemont AJ: Investigation of the role of IL-1 and TNF in matrix degradation in the intervertebral disc. Rheumatology (Oxford) 2008, 47:809-814.

22. Le Maitre CL, Hoyland JA, Freemont AJ: Interleukin-1 receptor antagonist delivered directly and by gene therapy inhibits matrix degradation in the intact degenerate human intervertebral disc: an in situ zymographic and gene therapy study. Arthritis Res Ther 2007, 9:R83.

23. Song RH, Tortorella MD, Malfait AM, Alston JT, Yang Z, Arner EC, Griggs DW: Aggrecan degradation in human articular cartilage explants is mediated by both ADAMTS-4 and ADAMTS-5. Arthritis Rheum 2007, 56:575-585.

24. Chiba K, Andersson GB, Masuda K, Thonar EJ: Metabolism of the extracellular matrix formed by intervertebral disc cells cultured in alginate. Spine 1997, 22:2885-2893.

25. NCBI: [http://www.ncbi.nlm.nih.gov/index.html].

26. Takara Bio Inc: [http://www.takara-bio.co.jp/].

27. Livak KJ, Schmittgen TD: Analysis of relative gene expression data using real-time quantitative PCR and the 2(-Delta Delta C(T)) method. Methods 2001, 25:402-408.

28. Masuda K, Aota $Y$, Muehleman C, Imai $Y$, Okuma M, Thonar EJ, Andersson GB, An HS: A novel rabbit model of mild, reproduc- 
ible disc degeneration by an anulus needle puncture: correlation between the degree of disc injury and radiological and histological appearances of disc degeneration. Spine 2005, 30:5-14.

29. Masuda K: Biological repair of the degenerated intervertebral disc by the injection of growth factors. Eur Spine J 2008, 17(suppl 4):441-451.

30. Seguin CA, Bojarski M, Pilliar RM, Roughley PJ, Kandel RA: Differential regulation of matrix degrading enzymes in a TNFalphainduced model of nucleus pulposus tissue degeneration. Matrix Biol 2006, 25:409-418.

31. Le Maitre CL, Hoyland JA, Freemont AJ: Catabolic cytokine expression in degenerate and herniated human intervertebral discs: IL-1beta and TNFalpha expression profile. Arthritis Res Ther 2007, 9:R77. 